\title{
Multi-slice Spiral Computed Tomography
}

National Cancer Institute

\section{Source}

National Cancer Institute. Multi-slice Spiral Computed Tomography. NCI Thesaurus.

Code C106545.

A type of spiral computed tomography that employs multiple rows of detectors, each capable of producing a simultaneous image (slice). 